\title{
Imaging in Patients with Various Pathologies Causing Facial Nervepalsy: Study of 70 Patients
}

\author{
Dr. K. P. Morwani ${ }^{1}$, Dr. Gaurav Ahluwalia ${ }^{2}$ \\ ${ }^{1}$ HOD, Dept. of Otorhinolaryngology, Dr. Balabhai Nanavati Hospital, Mumbai, India \\ ${ }^{2}$ PG Student, Dept. of Otorhinolaryngology, Dr. Balabhai Nanavati Hospital, Mumbai, India
}

\begin{abstract}
Facial nerve as we all knows is the main motor supply to the face which is believed to be the window to the soul. Paralysis of this nerve causes immense social and emotional trauma to the individual, which in most cases take precedence over the cosmetic and functional disfigurement. Many patients become reclusive and depressed; losing their confidence as well as identity. Hence, the timely treatment of this condition can bring about multifactorial benefits to the patient and a sense of immense gratification to the surgeon. In this thesis, I have briefly reviewed literatures in addition to briefly stressing on anatomy of this important structure as well as correlating the clinical, imaging and intraoperative Facial Nerve findings and its outcome in the management of this greatly traumatizing condition.
\end{abstract}

Keywords: Facial nerve, Bell's palsy, Acoustic neuroma, Facial nerve grafting, Facial Nerve mobilisation, Traumatic Facial Nerve palsy.

\section{Introduction}

Like most cranial nerves $(\mathrm{CN})$, Facial nerve contains motor, sensory and Parasympathetic fibers. Among its functions are the vital control of facial expression, taste to the anterior two-third of the tongue and salivary and lacrimal gland secretion.

Of all the cranial nerves, Facial Nerve is most susceptible to injury. The nerve travels a complex course through the temporal bone in proximity to various structures which are frequently the site of disease. Much of the nerve's susceptibility to paralysis can be attributed to these anatomical factors. Patients who suffer from facial paralysis experience not only functional consequences, but also the psychological impact of a change in self-image and impaired communication ability.

More than 40 different causes of facial paralysis are known, classified as idiopathic, congenital, traumatic, infections, neoplastic and metabolic. Of the various causes, $75 \%$ cases are of Bells palsy or secondary to trauma. In cases of facial paralysis, history and physical examination are very important. Diagnostic tests influence the choice of therapy and can improve outcome.

Successful management of the disease process that causes Facial Nerve dysfunction requires thorough knowledge of the pathophysiology of the disease process. Management of Facial Nerve dysfunction is individualized, and may include observation, administration of pharmacological agents, surgical interventions, physical therapy and psychological counselling.

Surgical Management of Facial Nerve disorders continues to be as controversial as it was in the days of Cawthorne and Ketter. Hence the degree of recovery also varies as per the modality and timing of treatment used. Keeping this in mind, this study aims at understanding the different causes of facial paralysis seen and diagnosed by computed tomography scan and co-relation of computed tomography findings with management.

\section{Aims \& Objectives}

1) Study of various pathologies causing Facial nerve palsy and degree of facial paresis / palsy.

2) Correlation of imaging findings with intra-operative findings and pathology suspected and management.

\section{Materials \& Methods}

1) Study Area - This study was carried out at Dr. Balabhai Nanavati hospital and Research centre, Mumbai, which is a tertiary care centre. It is a large Trust hospital located in the urban setup of Mumbai, catering to the needs of various socioeconomic strata of the local community having a capacity of 350 beds.

2) Study Population - Study included 70 patients with facial paresis / palsy who were referred to our institution for imaging study from August 2010 to August 2012 were included in the study. It included both sexes, all age groups, admitted, OPD patients as well as those referred to our radiology department from other centres.

3) Sample Size and Sample Technique - This is a prospective study of 70 patients with Facial nerveparesis or palsy who underwent imaging study in our hospital from August 2010 to August 2012.

4) Data Collection Technique and Tools - All the patients selected in the study entered into a structured proforma which included detailed history and clinical examination of the patient. Those patient were worked up for imaging studies and were treated medically or surgically and were followed up over period of six months.

A proper history was taken from the patient with the help of a Proforma. The proforma used is given at the end of this thesis. This was followed by the clinical examination and assessment of deformity. Investigations performed included routine investigations and special investigations like CT scan or MRI scan and audiometry.

After investigation the patient were treated medically or surgically as indicated. After informing the patient and obtaining prior written consent, the patient was subjected 


\section{International Journal of Science and Research (IJSR) \\ ISSN (Online): 2319-7064}

Index Copernicus Value (2013): 6.14 | Impact Factor (2014): 5.611

to surgery as and when indicated and conforming to well recognized and established modalities of treatment.

Post treatment the assessment of deformity and degree of improvement was assessed. Whatever the modality of treatment, the patients were followed up for a minimum period of six months when the improvement was again assessed.

5) Data Analysis - Data is tabulated and analyzed. Frequency and percentage tables are used to present the results. Analysis of quantitative variable is done with the help of Mean and represented with the help of appropriate charts and graphs.

\section{Inclusion Criteria}

1) Patients of all age group and both sexes.

2) Cases of postoperative facial palsy.

3) Patients presenting with recurrent episodes of facial palsy.

4) Cases of chronic Facial nervepalsy are also included in study.

5) Patients with Facial Nerve paresis / palsy who underwent CT / MRI imaging at Nanavati hospital between August 2010 and August 2012 are included in this study.

\section{Exclusion Criteria}

Patients who could not be followed up for the minimum stipulated time.

\section{Results}

The mean age of Facial Nerve paresis / palsy in our study was 43.5 years and adults seem to be affected predominantly. Males were affected commonly with ratio of 1.12: 1. Bell's palsy (24\%) Fig 7, 8 was the most common cause followed by Post surgical trauma (19\%). CSOM (including cholesteatoma and Skull base Osteomyelitis) (16\%), Head injury (14\%) Fig 1,2 Cerebro-Vascular accidents (14\%), CP Angle tumours (10\%) Fig 3,4,5,6 and Herpes Zoster (3\%), are the other causes. Bell's palsy was found more common in females than in males (1.4:1) and more in the adult age group (41-60yrs). Exposure to cold (25\%) was seen in a number of patients. Familial and recurrent Bells palsy is a rare feature. Head injury causing palsy is common in young adult males. Chronic suppurative otitis media with facial palsy is more common in females. $76 \%$ patients with Bells palsy had complete recovery with steroids while $70 \%$ of patients with trauma had complete recovery. All cases with surgical trauma had complete recovery. In CSOM with facial palsy the recovery was good if the nerve was not directly infiltrated by disease process.

\section{Conclusion}

Acute facial paralysis can result from various causes among which intratemporal facial palsy is most common. Among the various causes idiopathic Facial nerve palsy or Bells palsy is the most common cause. Bell's palsy is more common in adult males and so is post-surgical facial palsy. Infection and trauma is the other common cause.
Diagnosis of Facial nerve disorders can usually be made by a good clinical history, clinical examination supplemented by radiological investigations.

The majority of cases of Bells palsy and trauma recover with conservative treatment including steroids and physiotherapy. Other conditions like facial palsy secondary to CSOM especially when associated with cholesteatoma must be treated by surgery, but the prognosis may not always be satisfactory especially if the nerve is infiltrated. Herpes zoster causing facial palsy is rare.

Correlation between radiological imaging and surgical findings is better in conditions like tumours, trauma, CSOM.

\section{Recommendations}

1) Cases of Bells palsy which do not show any improvement in 2 months time should do imaging to rule out any other pathology in course of facial canal.

2) MRI with contrast is only imaging technique which shows inflammation of Facial Nerve which is a prerequisite for decompression. Besides it can also help in deciding which portion of Facial Nerve is to be decompressed.

3) Tumours/ pathology along vertical Fallopian canal are missed, if HRCT of temporal bone is done in slices of more than $0.6 \mathrm{~mm}$ or bone window is not available or if computed tomography scan is done only in axial plane. Haemangioma / small tumours along Fallopian canal can be missed on high resolution computed tomography with bone window. One has to do MRI with contrast with pathology.

4) Post head injury Facial Nerve palsy is secondary to fractures in temporal bone in $67 \%$ of cases only (Mark May). In one third patients the injury to Facial Nerve can be infratemporal/ intracranial portion and these can be diagnosed by MRI.

5) Small tumours of deeper lobe of parotid gland are missed on CT and can be picked up only on MRI with contrast.

6) There is not enough literature to emphasize the occurrence of neuroma of Facial Nerve in cerebellopontine angle angle. These will be picked up only by magnetic resonance imaging with contrast.

7) in absence if obvious fracture line of temporal bone in head injury cases, gross hematoma in middle ear and mastoid portion and haziness in geniculate ganglion area are pre-requisites for Facial Nerve decompression not emphasized in literature.

8) Recurrent Facial Nerve palsy/paresis is not always secondary to Bell's palsy. The small tumours of Fallopian canal occasionally do respond to heavy steroid therapy and recover completely and its mandatory to do MRI with contrast in such cases to exclude tumours.

9) Recurrent Bell's palsy must have MRI with contrast and if there is enhancement in course of Facial Nerve, one must perform Facial Nerve decompression even if patient has upto grade IV palsy.

10) Acute otitis media in neonates and infants with grade IV palsy should have immediate myringotomy and drainage of puss besides medical line of treatment. 


\section{International Journal of Science and Research (IJSR) \\ ISSN (Online): 2319-7064}

Index Copernicus Value (2013): 6.14 | Impact Factor (2014): 5.611

11) Cases of SBO with grade VI palsy not showing any evidence of recovery inspite of recovery from pain and discharge should be considered for Facial Nerve decompression.

12) Post-traumatic cases of Facial Nerve palsy should be explored as early as possible and in case they are explored late, to get better recovery one must excise end on neuromas on either sides more than adequately and also excise any length of fibrosed nerve. Grafting must be done between 2 ends of healthy nerve. The length of graft does not hamper the final recovery. We have done Facial Nerve grafting after 4.5 years and after 7 years of Facial Nerve palsy secondary to mastoid surgery with surprisingly good results.

\section{References}

[1] Philip AW, John SR. Facial paralysis. Ballengers otorhinolaryngology, Head and Neck Surgery,16th ed, Snow JB, Ballenger JJ. BC Decker Spain 2004:489-519

[2] Barry S, Mark M. Disorders of Facial nerve. ScottBrown's Otolaryngology'6th ed, Kerr AG, Bueth JB. Butterworth-Heinmann, London, 1997:3/24/1-3/24/38

[3] Robert AD. Tests of Facial nerve function. Otolaryngology and Head and Neck Surgery, 3rd ed, Cummings CW, Friederickson JM, Marker LA, Krauss C, Richardson MA, Schuller D. Mosby USA, 1998: $2757-2765$.

[4] Ravi NS, Bruce JG. Surgery of the Facial nerve. Surgery of the Ear, 5th ed, Michael EG, Aina JG. BC Decker, Canada, 2003:615-639

[5] Bruce JG, Jay TR. Intratemporal Facial nerve surgery. Otolaryngology and Head and Neck Surgery, 3rd ed, Cummings CW, Friederickson JM, Marker LA, Krauss C, Richardson MA, Schuller D. Mosby USA, 1998: $2785-2799$.

[6] Kai-Wen Z, Zi-Ting S. Microvascular decompression by the retrosigmoid approach for idiopathic hemifacial spasm: experience with 300 cases. Ann Otol Rhinol Laryngol 1995;104:610-612.

[7] John C, Mark. Perspectives in facial reanimation. The Facial Nerve,2nd ed, Mark M, Barry S. Thieme, New York,2000:551-570

[8] Raymond FG, Shuntaro S, Kazuyuki S. Three dimensional development of the Facial nerve path through the ear region in human embryos. Ann Otol Rhinol Laryngol 1994;101:395-403

[9] Mark M. Anatomy for the clinician. . The Facial Nerve,2nd ed, Mark M, Barry S. Thieme, New York,2000:19-56

[10] Richard RG. On the duality of the Facial nerve ganglion. Laryngoscope 199;108:1077-1086

[11] John MC, Gaily NJ. Patterns of Facial nerve synkinesis. Laryngoscope 1996;106:1491-1496

[12] Carol AB, Newton JC. Update on Facial nerve disorders. Otolaryngologic clinics of North America 1996;29:445454

\section{Figure Legends}

Fig (1) Post Traumatic Pre Operative Facial Nerve paresis

Fig (2) 6 months post operative Post Traumatic Facial nerve paresis

Fig (3) Pre Operative Acoustic Neuroma

Fig (4) 6 months Post Operative Acoustic

Fig (5) Pre Operative Glomus Jugulare

Fig (6) 10 months Post Operative Facial - Hypoglossal Anastomosis with Gold upper eyelid implant

Fig (7) Bell 's palsy Day 1

Fig (8) Bell's palsy 3 months

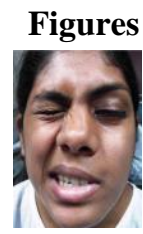

Fig (1) Post Traumatic Pre Operative Facial nerve paresis

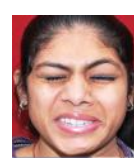

Fig (2) 6 months post operative Post Traumatic Facial nerve paresis

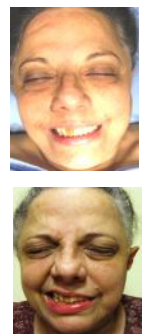

Fig (3) Pre Operative Acoustic Neuroma

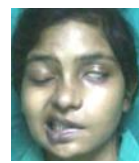

Fig (5) Pre Operative Glomus Jugulare

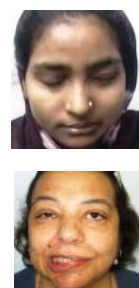

Fig (6) 10 months Post Operative Facial Hypoglossal Anastomosis with Gold upper eyelid implant

Fig (7) Bell's Palsy Day 1

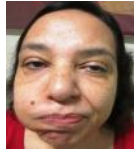

Fig (8) Bell's Palsy 3 months 